\title{
X-ray beam expansion by the application of re-entrant surface profiles to deformable bimorph mirrors
}

John Sutter, Simon Alcock, Yogesh Kashyap, loana Nistea, Hongchang Wang, et al.

John P. Sutter, Simon G. Alcock, Yogesh Kashyap, loana Nistea, Hongchang Wang, Kawal Sawhney, "X-ray beam expansion by the application of reentrant surface profiles to deformable bimorph mirrors," Proc. SPIE 9965, Adaptive X-Ray Optics IV, 99650E (8 September 2016); doi: 10.1117/12.2239116

Event: SPIE Optical Engineering + Applications, 2016, San Diego, California, United States 


\title{
$X$-ray beam expansion by the application of re-entrant surface profiles to deformable bimorph mirrors
}

\author{
John P. Sutter*, Simon G. Alcock, Yogesh Kashyap, Ioana Nistea, Hongchang Wang, Kawal \\ Sawhney \\ Diamond Light Source, Harwell Science and Innovation Campus, Chilton, Didcot OX11 9RE, \\ United Kingdom
}

\begin{abstract}
Deformable, piezo bimorph mirrors are often used to expand X-ray beams to a continuous range of sizes. However, optical polishing errors present on all X-ray mirrors introduce striations into the reflected beam. To counteract them, reentrant surface modifications with alternating concave and convex curvature have been proposed and applied to mirrors of fixed shape or bimorph mirrors. For the latter, a new method of constructing re-entrant surface modifications on segments of unequal length is described. This allows the re-entrant modification required for a desired beam size at the focal point to be matched to the bimorph mirror's polishing errors, thus reducing the voltage variations. Optical profilometry using the Diamond-NOM showed that a 5-segment and a 7-segment modification could be suitably applied to a deformable bimorph mirror. X-ray tests showed that striations caused by the 5 -segment modification in the beam at the focus are concentrated at the beam edges, while the beam center is left clear. This is in contrast to simple defocusing, in which a strong side shoulder appears. The 7-segment modification produces a pattern of evenly spaced striations. The intensity spikes seen with the re-entrant modifications are caused chiefly by the finite curvature of the mirror at the turning points. The question of whether deformable bimorph mirrors with different piezo response functions could sharpen the curvature changes will be investigated. Optimal modifications of continuous curvature, which could more realistically be applied, will be sought.
\end{abstract}

Keywords: X-ray, mirror, bimorph, surface, profile

\section{INTRODUCTION}

In recent years beamline scientists at synchrotron light sources have expressed their desire for the ability to shape the transverse profile of X-ray beams. Often they wish not for the tightest possible focus, but for a uniform beam ("tophat") of a size that matches their sample, typically from below $1 \mu \mathrm{m}$ up to $100 \mu \mathrm{m}$. A program to develop X-ray beam shaping techniques is under way at the Diamond Light Source, the United Kingdom's national synchrotron facility. Mirrors are being investigated for this purpose, but surface figure errors continue to limit their performance, even though with current technology errors below $5 \mathrm{~nm}$ rms are routine and state of the art values go as low as $0.1 \mathrm{~nm}$ rms.

A mirror designed for beam shaping must have a variable surface. Active mirrors with deformable reflecting surfaces would allow the beam size to be varied within a continuous range. Designs of deformable mirrors that could remove the effect of atmospheric turbulence from astronomical imaging go back to $1953^{1}$, but in the synchrotron community, bimorph mirrors like those common in astronomy began to appear only in the late 1990's with the advent of thirdgeneration synchrotrons ${ }^{2-4}$, and were designed with stable beam focusing over hours or days in mind. Unlike astronomical bimorph arrays, bimorph mirrors for synchrotron X-rays focus in one direction only, as ellipsoidal mirrors with two-dimensional focusing are difficult to fabricate with the required precision, and are typically restricted by their construction to 8-16 actuators rather than the hundreds or even thousands in astronomical bimorph mirror arrays. However, this enforced simplicity of bimorph mirrors for synchrotron X-rays makes the actuator settings for ideal deformations relatively simple to calculate. In the following, all purposely introduced surface deformations will run along the mirror's length only; the shape of the mirror surface across its width will be left unchanged. All modifications will be added to the ideal elliptical arc to which the mirror would be shaped along its length for perfect one-dimensional focusing of a point source to a point image.

*john.sutter@diamond.ac.uk; phone 441235 778626; fax 44 1235 778784; www.diamond.ac.uk 
Geometrical optics has been used in previous work and will also be used here. This is justified theoretically for X-rays of wavelength $\lambda$ by the treatment of Laundy, Alianelli et $\mathrm{al}^{5}$. Suppose that a periodic modulation of wavelength $\Lambda$ is applied to the surface of a focusing mirror. Let $q$ be the distance from the mirror to the focal plane and let $\theta$ be the grazing angle of incidence at the center of the mirror. The modulation behaves like a diffraction grating producing fringes with a spacing of $q \lambda /(\Lambda \sin \theta)$ in the focal plane. If the spacing between the fringes is much smaller than the focal spot size $\Sigma$ produced by the unmodified mirror surface, the fringes overlap, making geometrical optics valid for relating the beam profile at the focus to the mirror surface's figure. This condition is met if $\Lambda>>\lambda \lambda /(\Sigma \sin \theta)$. In the following paper, $q$ is approximately $0.4 \mathrm{~m}, \lambda$ is either $1.35 \AA$ or $1.55 \AA, \Sigma$ has been measured experimentally at approximately $1 \mu \mathrm{m}$, and $\theta$ is approximately $3 \mathrm{mrad}$. The surface modulation's wavelength $\Lambda$ must therefore be much greater than $18 \mathrm{~mm}$ or $20.67 \mathrm{~mm}$, depending on $\lambda$. The surface modifications of this paper are not periodic, but will not vary over scales smaller than $30 \mathrm{~mm}$. Therefore, undesirable wavelength-dependent diffraction effects may be neglected. The use of geometrical optics is also justified experimentally by its ability to generate theoretical beam profiles in good agreement with knifeedge scans of X-ray beams reflected by a mirror of known surface error at the undulator beamline I02 of Diamond Light Source ${ }^{6}$.

The first ideal mirror surface modifications for beam shaping to specific profiles were calculated by Spiga et $\mathrm{al}^{7}$. Although they assumed positive curvature along the entire length of the mirror ("defocusing"), they also pointed out that "re-entrant" surface modifications, which have positive curvature on some sections and negative on others, can also shape the beam at the mirror focus. In the absence of figure errors, all would be equally effective, but Nicolas and García $^{8}$ showed that polishing errors introduce striations into the reflected beam. The striations of beam reflected by a defocused mirror are spread out across the beam's full width, but ray traces in Laundy, Alianelli et $\mathrm{al}^{5}$ predict that striations of beam reflected by different segments of a re-entrant modification are superimposed and thus average out, smoothing the beam profile.

The first experimental test of re-entrant surface modifications was performed by Laundy, Sawhney et $\mathrm{al}^{9}$. Three lanes were polished into a mirror of fixed figure. One was optimized for beam focusing to below $1 \mu \mathrm{m}$, while the other two were made with re-entrant surfaces of four equal segments, one for $2 \mu \mathrm{m}$ and the other for $10 \mu \mathrm{m}$ beam at the mirror focus. Deformable piezo bimorph mirrors, by contrast, can adjust the beam shape and size at the focus through a continuous range, but they have two limitations. Firstly, the piezos are least able to correct polishing errors at the junctions between adjacent electrodes. Secondly, the voltage difference between adjacent electrodes cannot exceed $500 \mathrm{~V}$ without risk of damage to the mirror. As a result, re-entrant surface modifications applied to bimorph mirrors must have the junctions between adjacent segments ("breakpoints") on the junctions between adjacent electrodes. The segments of the re-entrant modification need not, however, be of equal length. Using this freedom, one can choose a reentrant modification that matches the mirror's figure errors when all voltages are zero, minimizing the voltages that must be applied, by extending one or more segments across multiple electrodes.

In the following, re-entrant modifications with a variable number of segments of arbitrary length will be calculated under the assumption that the X-ray mirror is uniformly illuminated along its length. Two modifications, one with five segments and the other with seven, will be calculated for a $4 \mu \mathrm{m}$ wide beam at the mirror's focus and applied to a bimorph mirror "super-polished" to exceptionally low figure error. Both ex situ and in situ measurements will show how nearly the bimorph mirror actually matches each. The smoothest expanded beam profiles will be displayed. Ray traces by Sutter, Alcock, Kashyap et a ${ }^{10}$ attribute the spikes in these profiles to undesirable rounding of the curvature of the surface modification at the breakpoints. Geometrical optics explains this as the piling up of rays reflected from regions of the mirror where the curvature of the modification is close to zero; that is, the mirror's curvature is that of the ideal ellipse. The possibility of reducing these spikes by sharpening the switch in curvature at the breakpoints will be examined using other bimorph mirrors of different design. Suggestions will be made on applying surface modifications of continuous curvature, which in practice are all that is possible for deformable bimorph mirrors, while avoiding excessive beam striation.

\section{PRINCIPLES}

This method generalizes the one reported by Laundy, Alianelli et $\mathrm{al}^{5}$ for generating re-entrant surface modifications on segments of equal length if the mirror is uniformly illuminated. The height of the modification and its first derivative (the slope) are continuous, but its second derivative (the curvature) is made discontinuous at the breakpoints so that the curvature has no zeros to cause spikes in the reflected beam. The X-ray beam at the mirror's focus is to be expanded to a width $B$. The mirror has length $L$, and a surface modification of $n$ segments is to be added to its original elliptical surface. 
$q$ is the distance from the center of the mirror to the focus, and $\theta$ is the grazing angle of the incident beam at the mirror's center. $x$ is the coordinate along the length of the mirror, with $x=0$ at the center and positive $x$ toward the focus.

The breakpoints $x_{j}$, where $j=0, \ldots, n$, are defined such that

$$
-\frac{L}{2}=x_{0}, x_{j}<x_{j+1}, x_{n}<+\frac{L}{2} .
$$

For each segment $i=1, \ldots, n$, the quantity $S_{i}$ is defined:

$$
S_{1}=\frac{B}{2\left(x_{1}-x_{0}\right)}, S_{i}=\frac{B}{\left(x_{i}-x_{i-1}\right)}, S_{n}=\frac{B}{2\left(x_{n}-x_{n-1}\right)} \text { for } i=2, \ldots, n-1 .
$$

Laundy, Alianelli et $\mathrm{al}^{5}$ show how energy conservation results in a separable differential equation of which the solution is the height $A_{i}(x)$ of the $i$ th segment of the surface modification from $x_{i-1}$ to $x_{i}$ :

$$
A_{i}(x)=\frac{1}{2 \cos \theta}\left(-\frac{C_{1 i}}{\cos \theta} \ln (q-x \cos \theta)+(-1)^{i} S_{i} x+C_{2 i}\right) .
$$

Its derivative, the slope $A_{i}^{\prime}(x)$ on the $i$ th segment from $x_{i-1}$ to $x_{i}$, is thus

$$
A_{1}^{\prime}(x)=\frac{1}{2 \cos \theta}\left(\frac{C_{1 i}}{q-x \cos \theta}+(-1)^{i} S_{i}\right),
$$

where the $2 n$ constants of integration $C_{1 i}$ and $C_{2 i}$ are determined by the boundary conditions:

$$
\begin{gathered}
A_{1}\left(x=-\frac{L}{2}\right)=0 \\
A_{n}\left(x=+\frac{L}{2}\right)=0 \\
A_{k}\left(x=x_{k}\right)=A_{k+1}\left(x=x_{k}\right) \\
A_{k}^{\prime}\left(x=x_{k}\right)=A_{k+1}^{\prime}\left(x=x_{k}\right),
\end{gathered}
$$

where $k=1, \ldots, n-1$. Thus there are $2 n$ equations to determine the $2 n$ constants of integration, calculated using MATLAB $^{11}$. Note that Eq. (4) can be expanded in a Taylor series about $x_{i}$. Only the zeroth and first order terms in $\left(x-x_{i}\right)$ are significant for the mirror in this paper since $L / 2<<q$. Therefore, slope modifications appear piecewise linear, with their local maxima and minima at the breakpoints ${ }^{5,10}$.

\section{PROCEDURE}

\subsection{Bimorph mirror}

The bimorph mirror is an uncoated $150 \mathrm{~mm}$ long, deformable piezo bimorph mirror with a silica substrate and eight electrodes. The central $115 \mathrm{~mm}$ of the substrate was "super-polished" with elastic emission machining ${ }^{12}$ by JTEC (Japan) to an elliptical cylinder of source-mirror distance $41.5 \mathrm{~m}$, mirror-image distance $0.4 \mathrm{~m}$, and grazing incidence angle $3 \mathrm{mrad}$. Thales-SESO (France) attached the piezoelectric elements to the substrate. Over more than five years of operation, the mirror's surface profile and voltage responses have remained stable ${ }^{13}$.

\subsection{Mirror figure measurements}

As even figure errors of a few nanometers disturb the profile of the beam reflected by the mirror, metrological techniques of correspondingly high accuracy are required to detect them. Ex situ metrology was performed on the bimorph mirror using the Diamond-NOM, a non-contact slope-measuring profiler that measures angular surface deviations with an 
accuracy better than $50 \mathrm{nrad}^{14}$. This device is inside the Diamond Light Source Optics and Metrology laboratory, a Class 10,000 (ISO7) cleanroom. For in situ metrology, performed while exposing the mirror to X-rays, X-ray speckle tracking was applied ${ }^{15}$. A sandpaper diffuser was placed upstream from the mirror in order to introduce a speckle pattern into the reflected beam. As the diffuser was scanned transversely to the incident beam, cross-correlations between the speckle patterns at different diffuser positions were used to determine the mirror's slope error (first derivative of its figure error).

\subsection{X-ray beam profile measurements}

The mirror was examined with X-rays at the Diamond Light Source Versatile Test Beamline B16 ${ }^{16}$ using the setup shown in Fig. 1. X-rays with a photon energy of $9200 \mathrm{eV}$ were selected by a Si $\left(\begin{array}{lll}1 & 1 & 1\end{array}\right)$ double crystal monochromator (DCM). Slits downstream of the DCM selected a $0.03 \mathrm{~mm}$ (horizontal) $\times 0.3 \mathrm{~mm}$ (vertical) section of the X-ray beam produced by the bending magnet source. The small vertical slit size justifies the assumption of uniform illumination of the mirror. The EEM bimorph mirror was oriented for upward deflection inside a closed chamber that was continuously flushed with $\mathrm{N}_{2}$ gas at a rate of $1 \mathrm{~L} / \mathrm{min}$ to prevent the generation of reactive hydrocarbons which can contaminate the optical surface. Kapton windows allowed the X-rays to enter and exit the sealed chamber.

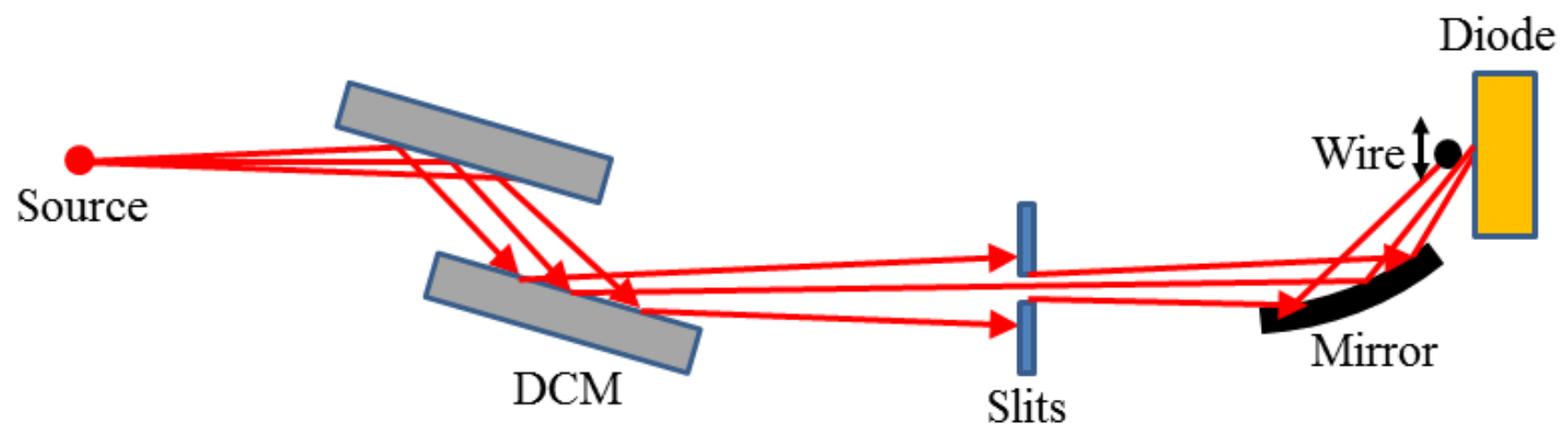

Figure 1: Schematic of the experimental setup used to measure the reflected X-ray beam profiles in the vicinity of the focus of the bimorph mirror at the Versatile Test Beamline B16 of Diamond Light Source. The figure is not to scale. From Sutter, Alcock, Kashyap et $\mathrm{al}^{10}$.

The transverse profile of the reflected X-ray beam in the vicinity of the mirror's focus were measured by knife-edge scans using a horizontal gold wire $200 \mu \mathrm{m}$ in diameter. The narrow horizontal width of the slits ensures that any striations are not blurred by sagittal slope errors on the mirror or by accidental inclination of the gold wire. The X-ray intensity passing by the gold wire was measured by a PIPS detector with a current amplifier.

The knife-edge scans taken while the re-entrant modifications were applied to the mirror were compared with a scan taken when the mirror was simply defocused. The latter scan was taken using X-rays of $8000 \mathrm{eV}$, but with all other experimental conditions the same except for possible slight variations in temperature and mounting forces. Despite the difference in photon energy between the older and the newer scans, the main features in the beam profiles can be explained to a good approximation by geometrical theory, in which the photon energy is not important ${ }^{10}$.

\section{RESULTS}

\subsection{Selection of re-entrant surface modifications for bimorph mirror}

The re-entrant surface modifications applied to the bimorph mirror were chosen by inspection of its piezo response functions (PRF) and of its figure when all voltages were set to $0 \mathrm{~V}$. Each electrode of the bimorph mirror has a PRF, which shows how a specific voltage increment applied to that electrode changes the mirror's overall figure. The PRFs may be expressed in terms of height or slope, the latter being the derivative of the former. They were determined by using the Diamond-NOM according to the procedure described by Hignette et $\mathrm{al}^{17}$ and by Sutter, Alcock \& Sawhney ${ }^{18}$, beginning with all voltages set to $0 \mathrm{~V}$ and using a $+200 \mathrm{~V}$ increment. Experience has shown that the mirror figure's response to voltage changes on the electrodes is highly linear; therefore, voltage optimization was performed by the linear algebra method of least squares minimization. The PRFs are shown in Fig. 2 in terms of slope because the region of the mirror influenced by each electrode is then obvious. The mirror's slope error when all voltages are at $0 \mathrm{~V}$ is also displayed there. This is equal to the slope measured by the Diamond-NOM minus the ideal focusing ellipse. 
By placing the breakpoints on the electrode junctions, one can approach as closely as possible the ideal discontinuous change in the sign of the curvature there, thus limiting intensity spikes in the beam at the focus. Experimentally, the positions of the electrode junctions were defined as the midpoints between the centers of adjacent PRFs in Fig. 2. The first breakpoints are placed at those junctions where the derivative of the slope error at $0 \mathrm{~V}$ most abruptly switches sign, in this case between electrodes 2 and 3 and between electrodes 6 and 7. This by itself produces a three-segment modification, which is displayed in Fig. 3(a) along with one five-segment modification generated by adding two more breakpoints and one seven-segment modification generated by adding four more breakpoints. Figure 3(b) shows a simple defocusing of the mirror. All modifications are designed to expand the beam size at the mirror's focus to $4 \mu \mathrm{m}$.

\subsection{Verification of surface modifications with the Diamond-NOM}

Figure 4 shows the three-segment modification. The list of voltages in Fig. 4(a) confirms that all voltage differences between adjacent electrodes remain within the $500 \mathrm{~V}$ limit. Figure 4(b) shows the slope error, the mismatch between the ideal re-entrant modification and the measured one. Figure 5 shows the same information for the five-segment modification with some small voltage changes made to keep the voltage difference within the $500 \mathrm{~V}$ limit. Figure 6 shows the seven-segment modification, with somewhat larger voltage changes made to keep the voltage difference within the design limit. Agreement of the final measured surface modifications is good throughout: $0.19 \mu$ rad for three segments, $0.29 \mu \mathrm{rad}$ for five segments, and $0.44 \mu \mathrm{rad}$ for seven segments. As expected, the curvature around the breakpoints is not in fact truly discontinuous; some rounding of the slope is always observed. A flattening of the measured slope modification is visible in the seven-segment modification at the junction of electrodes 4 and 5 , and to a lesser extent at the center of the mirror when the three-segment modification is applied. This "imprint" effect, caused by the electrodes' finite width, was observed by Alcock, Sutter et $\mathrm{al}^{19}$ and discussed more fully in Sutter, Alcock, Rust et $\mathrm{al}^{6}$.

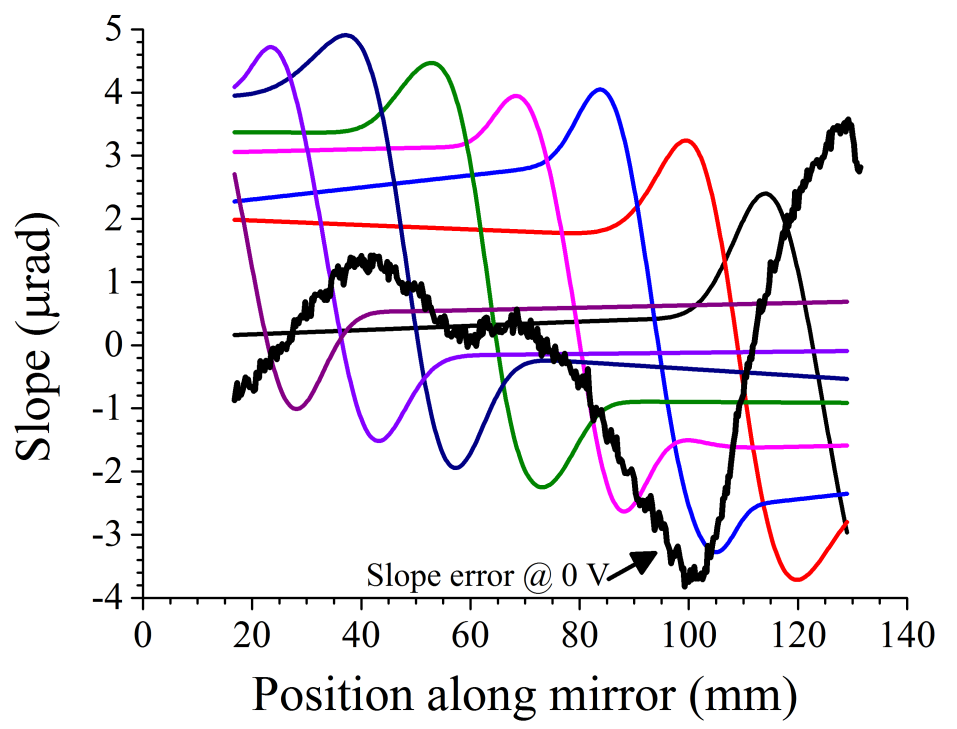

Figure 2: Piezo response functions displayed as slope changes scaled linearly to a voltage change of $500 \mathrm{~V}$ for comparison with the thick black curve that shows the mirror's slope error when all electrodes are at 0 V. From Sutter, Alcock, Kashyap et $\mathrm{al}^{10}$.

\subsection{X-ray measurements of surface modifications and beam profiles at focus}

Figures 7(a) and 7(b) compare, respectively, the theoretical five-segment and seven-segment surface modifications with measurements on the Diamond-NOM and with the results of X-ray speckle tracking at B16. They are not exactly in agreement and indeed were not expected to be, partly because X-ray speckle tracking is still a technique under development, and partly because the mounting forces and temperature variations to which the mirror was subjected were less well controlled at B16 than inside the cleanroom. Nonetheless, both the Diamond-NOM and the X-ray speckle tracking agree exactly in the number of segments and approximately in the positions of the breakpoints. The main disagreements among the three plots in each graph are observed close to the breakpoints. The measured curvature there is significantly rounded while the theoretical curvature changes sign abruptly, and the level of the slope at the 
breakpoints varies significantly. The imprint effect observed in the central segment of the seven-segment modification on the Diamond-NOM is also visible, though less pronounced, in the X-ray speckle tracking data.
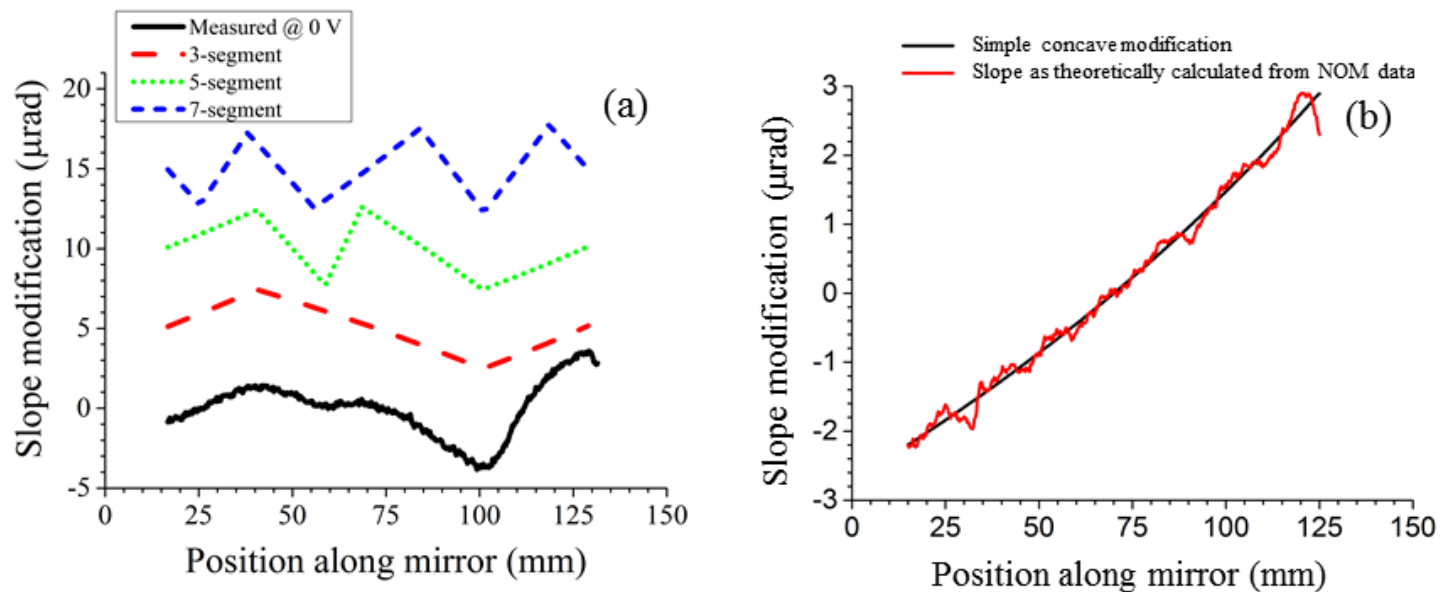

Figure 3: (a) Bottom to top: mirror slope error at $0 \mathrm{~V}$, then theoretical three-, five-, and seven-segment surface modifications approximating the slope error at $0 \mathrm{~V}$. Each plot has been shifted upward by $5 \mu$ rad from the one before for visibility. (b) Theoretical simple concave surface modification with expected best approximation achieved by mirror as calculated from its slope error at $0 \mathrm{~V}$ and its measured PRFs. Intended beam size at focus is $4 \mu \mathrm{m}$ for all cases. From Sutter, Alcock, Kashyap et $\mathrm{al}^{10}$.
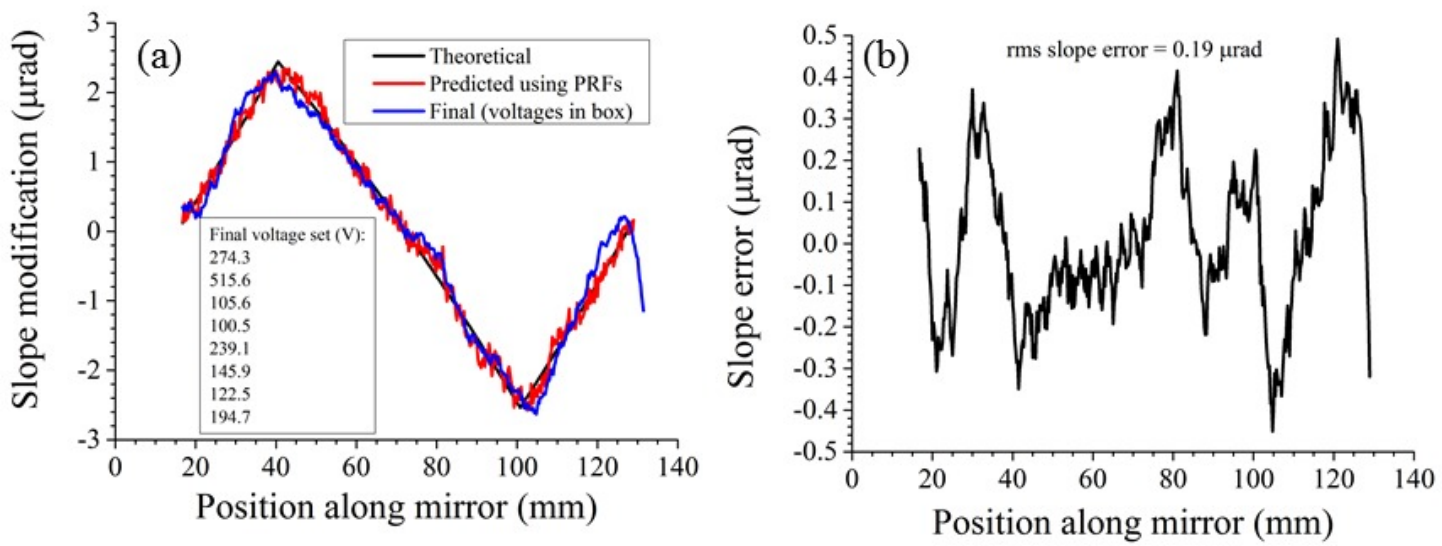

Figure 4: (a) Theoretical, predicted, and measured three-segment surface modification. (b) Slope error (deviation of measured surface modification from theoretical one). From Sutter, Alcock, Kashyap et $\mathrm{al}^{10}$.

Figure 8 compares typical beam profiles obtained by knife-edge scans at the mirror's focus when the seven-segment modification, the five-segment modification, and a simple concave modification were applied to the mirror. The sevensegment modification produces a series of spikes that are dispersed throughout the beam profile by the quite different levels of the slope at different breakpoints. However, these spikes appear at evenly spaced intervals, and the two central spikes are of similar strength and lie on a similar background level. The five-segment modification, by contrast, is dominated by the intense spikes at both edges. These hot edges are typical of slope modifications of continuous curvature such as sinusoids, because near the extrema of the slope, the position of the reflected ray on the detector varies minimally with the position of the incident ray. However, the center of the beam has considerably less striation. The profile produced by a simple concave modification has a strong side shoulder and shows a main peak that is breaking up into two equal parts even at this small $(5 \mu \mathrm{m})$ level of beam expansion. These arise from regions of zero curvature at random positions on the mirror. The positions of these regions depend on the polishing errors and would therefore be different for every mirror. 

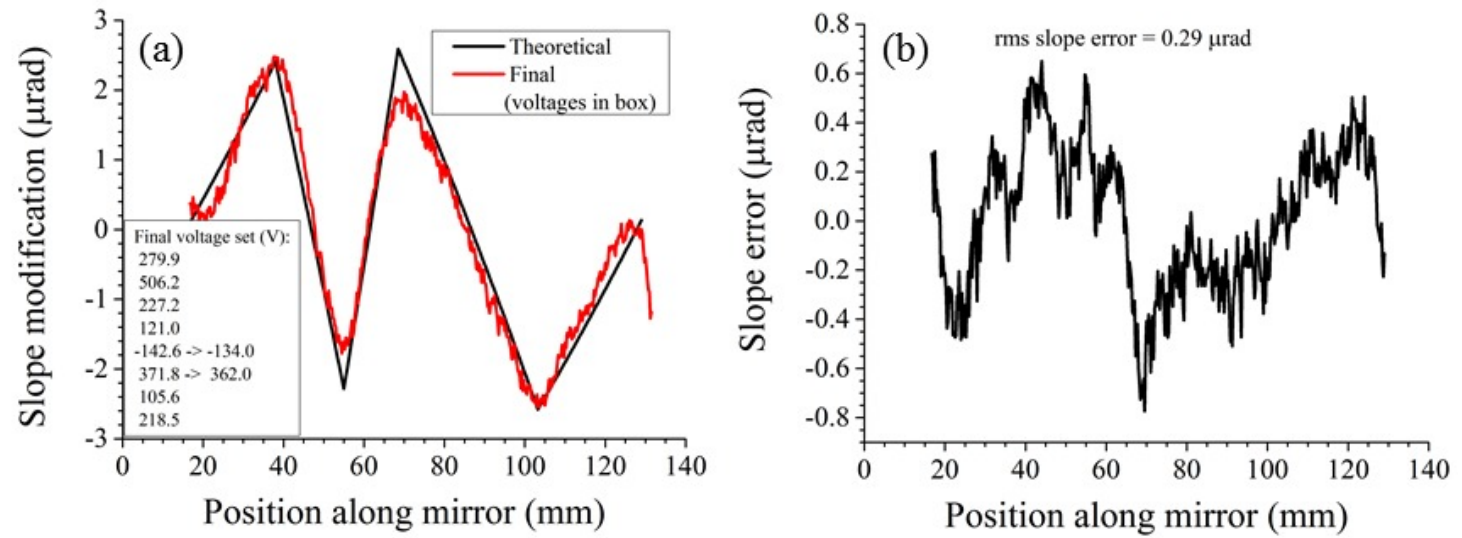

Figure 5: (a) Theoretical and measured five-segment surface modification. (b) Slope error. Arrows in the voltage list in (a) show where ideal voltages had to be compromised to keep voltage differentials below the $500 \mathrm{~V}$ limit. From Sutter, Alcock, Kashyap et $\mathrm{al}^{10}$.
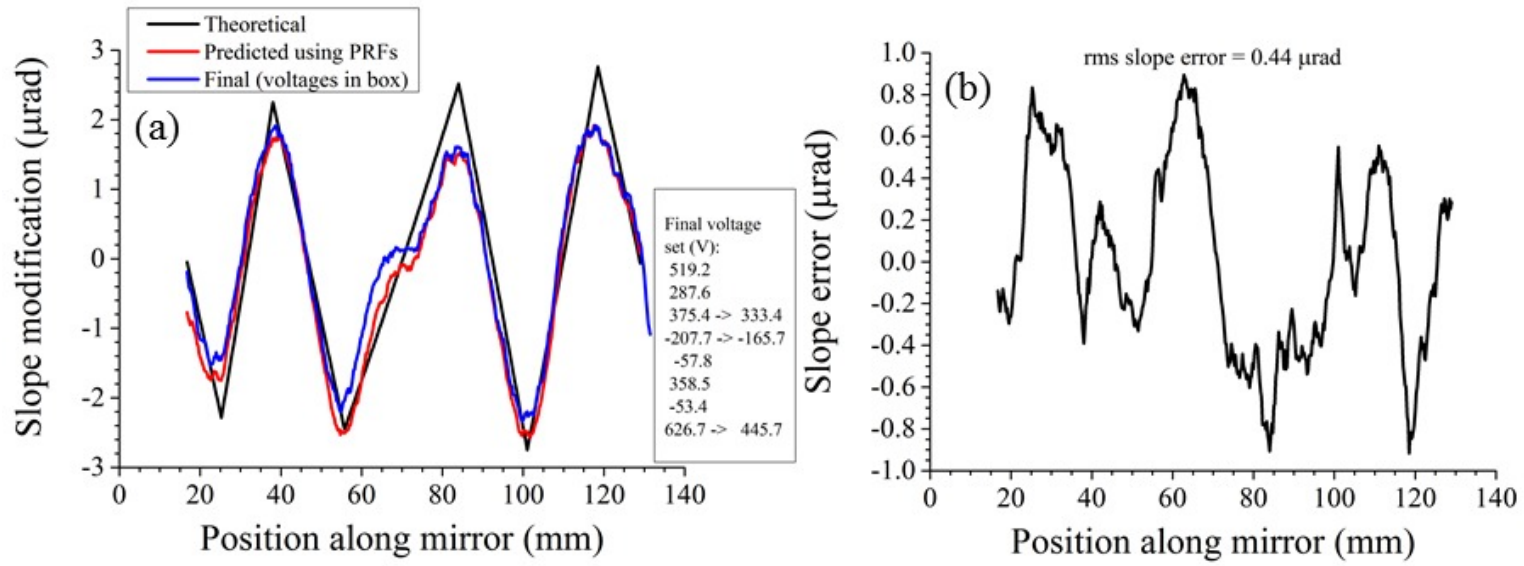

Figure 6: (a) Theoretical, predicted, and measured seven-segment modification. (b) Slope error. Arrows in the voltage list in (a) show where the voltages had to be shifted away from their theoretically optimal values to keep the voltage differentials below the $500 \mathrm{~V}$ limit. From Sutter, Alcock, Kashyap et $\mathrm{al}^{10}$.
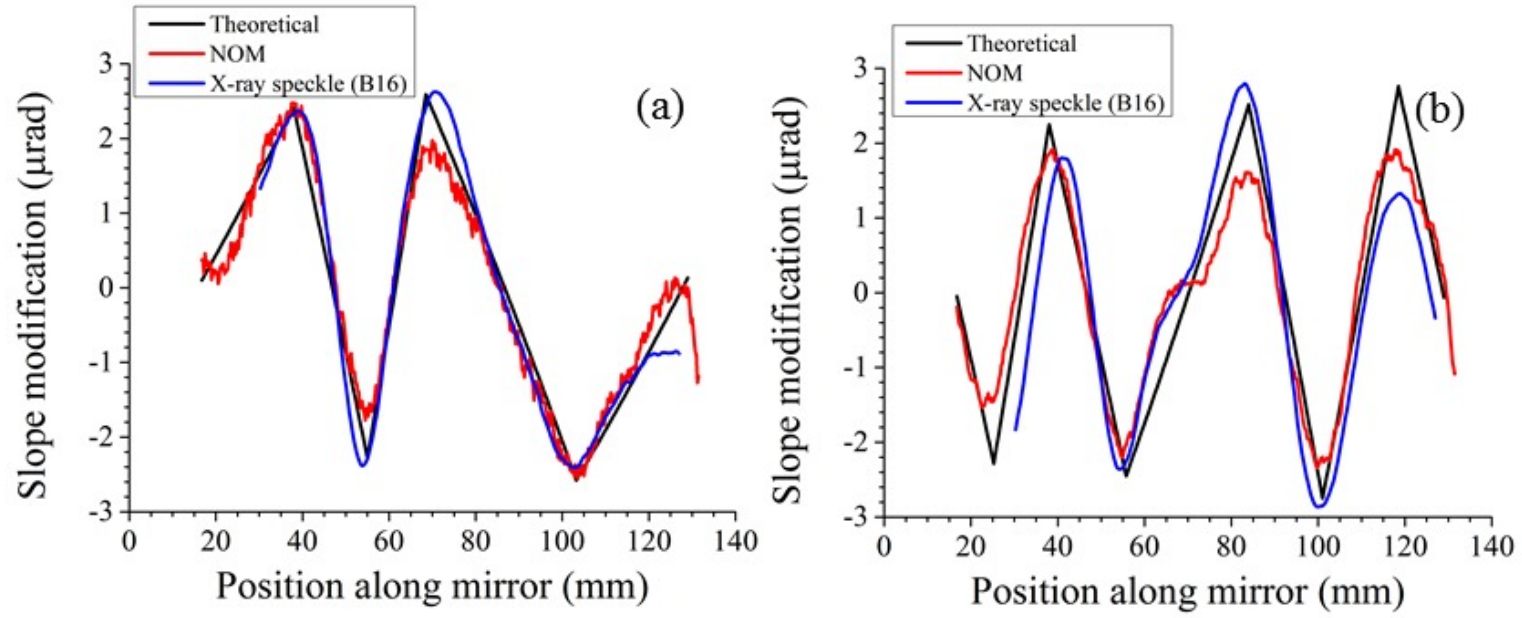

Figure 7: (a) 5-segment and (b) 7-segment surface modifications: comparisons of theory with measurements by DiamondNOM and by X-ray speckle tracking at B16. The theoretical modifications have sharp changes in curvature at their breakpoints. The data from B16 are represented by the smooth curve with the rounded extrema. From Sutter, Alcock, Kashyap et $\mathrm{al}^{10}$. 

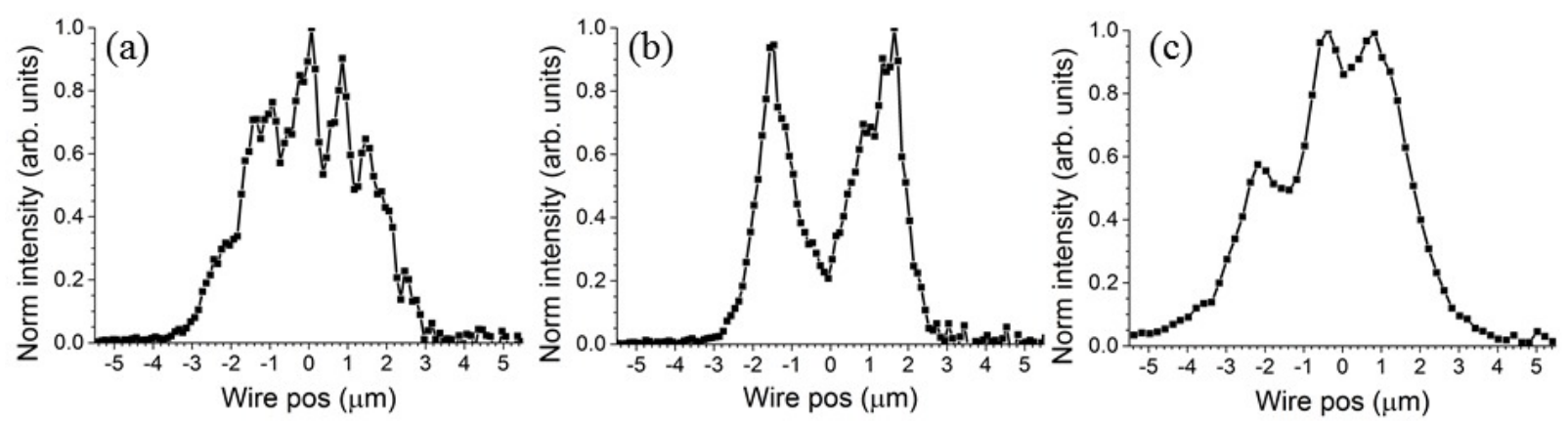

Figure 8: Beam profiles at mirror focus. (a) Seven-segment for $4 \mu \mathrm{m}$ beam (b) Five-segment for $4 \mu \mathrm{m}$ beam (c) Simple concave for $5 \mu \mathrm{m}$ beam: Maximum experimental intensity normalized to unity for easy comparison of plots. From Sutter, Alcock, Kashyap et $\mathrm{al}^{10}$.

\section{FURTHER DISCUSSION}

\subsection{Examination of mirrors with different piezo response functions}

Each bimorph mirror used at Diamond Light Source has piezo response functions of different characteristic shape. Their differences are most obvious when one examines them in terms of their slope. As stated before, the mirror used in the experiments described above has the piezo response functions of Fig. 2. The strong overshoot and undershoot on the edges of each PRF's central region distinguish this mirror from two others that will be examined here. Also significant is the lack of overlap between the central regions of the PRFs of adjacent electrodes. Note that each PRF reaches its minimum slope at approximately the position where the next PRF reaches its maximum slope. In the following section, the bimorph mirrors were all assembled by Thales-SESO (France).

One of the older bimorph mirrors at Diamond Light Source, the vertical focusing mirror at beamline I02, is $50 \mathrm{~mm}$ wide and has 8 electrodes over a length of $600 \mathrm{~mm}$. Figure 9(a) shows two typical PRFs of this mirror as measured with Xrays on the beamline. Note the absence of overshoot or undershoot and also the clear separation between them. Figure 9(b) shows the calculated result of adding the first PRF and subtracting the second PRF, thus simulating a real re-entrant surface modification. The breakpoint for the mirror at $\mathrm{I} 02$ has a sharper change in curvature than is seen at the breakpoints in Figs. 5(a) and 6(a), thus indicating that the overshoot and undershoot around the PRFs of this article's test mirror are causing some of the observed rounding.

At the other extreme is one of the newest bimorph mirrors to be installed at Diamond Light Source, the vertical microfocusing mirror of beamline I24. This mirror is $34 \mathrm{~mm}$ wide and has 16 electrodes along its $240 \mathrm{~mm}$ length. The PRFs of this mirror were measured both on the Diamond-NOM and with X-rays on the beamline. Because the measurement noise was considerable, an idealized model was developed in which each PRF was replaced by an error function of approximately the same width, strength, and position (Fig. 10(a)). The strong overlap between adjacent PRFs has been preserved. Only 14 electrodes are modeled here because the PRFs of the last two electrodes of the real mirror were cut off at the end. Nevertheless, this is sufficient to show that the strong overlap of the PRFs makes the achievement of a re-entrant surface modification impossible, at least by the expected method of adding the PRFs together with alternating sign (Fig. 10(b)). Figure 10(c) shows that the central region of this bimorph has only a small oscillating slope variation probably due to the imprint effect.

The reasons for the great variation of the PRFs' shape and overlap among these three mirrors are not fully understood. However, it should be mentioned that the test mirror of this article and the mirror used at $\mathrm{I} 02$ both belong to the first generation of bimorphs, in which the piezoelectric elements are sandwiched between an upper and a lower substrate ${ }^{2}$. On the other hand, the mirror installed at I24 is one of the first examples of the second generation of bimorphs, in which the piezoelectric elements are attached to the sides of the substrate ${ }^{20}$. The dependence of the PRFs on the construction of a bimorph will be an important topic for future studies. 


\subsection{Re-entrant modifications of continuous curvature}

Because in practice a deformable bimorph mirror cannot have a discontinuity in its curvature at any point along its length, it would be helpful to know how the features of surface modifications of continuous curvature might be chosen to minimize the striation in the reflected beam at the focus. One hint comes from the intense hot edges produced by the five-segment modification, which are shown in Fig. 8(b). The X-ray speckle tracking data in Fig. 7(a) show that the slope maxima are all at nearly the same level, as are the slope minima. Therefore, one could consider breaking up the hot edges by purposefully giving randomly varying values to the slopes at the breakpoints. To keep the beam's center clear, one could limit the random values to an upper band and a lower band, as shown in Fig. 11. This is in fact what occurred accidentally in the measured seven-segment modifications of Fig. 7(b).

A second unacceptable feature of beam profiles produced by sinusoidal modifications is that the hot edges have tails extending a long distance toward the center of the beam. These arise from the gradual reduction in the curvature of the sinusoidal modification as the slope extrema are approached. To avoid this problem, the slope of the modification's segments should follow the form given in Eq. (4) as closely as possible over as great a range as possible. This ensures an even spread of illumination across the reflected beam profile at the focus. For short mirrors such as that of this paper, for which Eq. (4) can be well approximated by a linear function, this condition can be met reasonably well by the PRFs of Fig. 2.
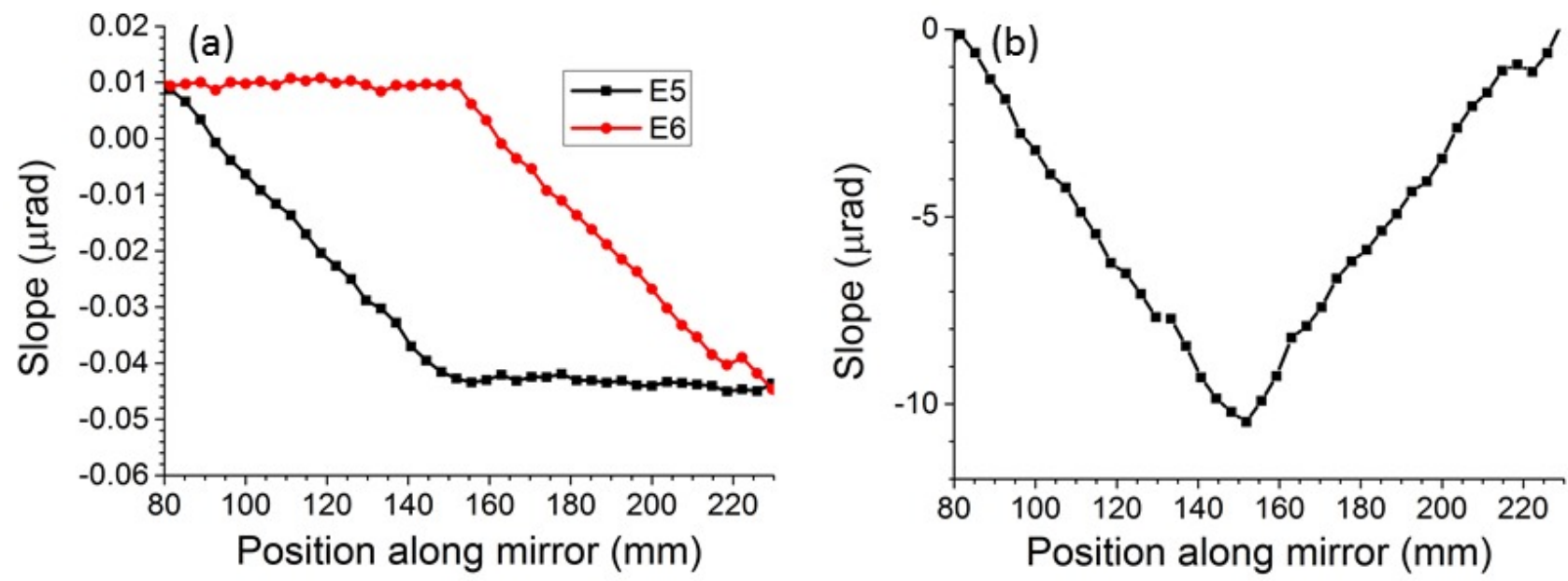

Figure 9: (a) Two typical adjacent PRFs E5 and E6 of the vertical focusing mirror of the Diamond Light Source beamline I02 (electrodes 5 and 6 of 8) given per volt. Measured using X-rays at the beamline. (b) $200 \times($ E6-E5), simulating the application of $200 \mathrm{~V}$ on electrode 5 and $-200 \mathrm{~V}$ on electrode 6 to produce a re-entrant surface modification.
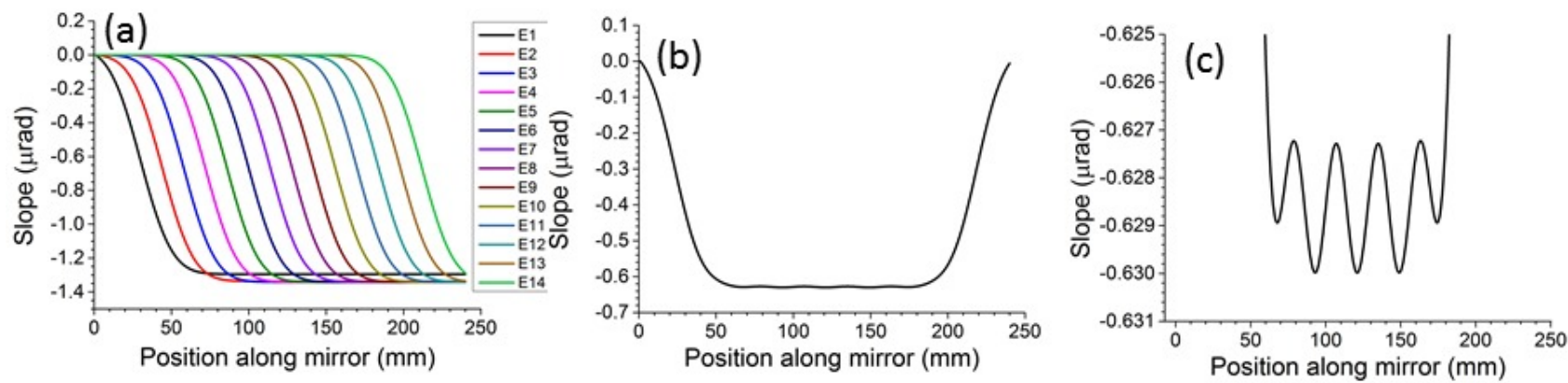

Figure 10: (a) Simplified theoretical model of PRFs of the vertical microfocusing mirror installed on the Diamond Light Source beamline I24. Based on measurements with the Diamond-NOM but showing only the first 14 of the 16 electrodes. Voltage increment is $200 \mathrm{~V}$. (b) Addition of PRFs in (a) with alternating signs. (c) Enlargement of vertical scale in (b) to show the imprint effect. 


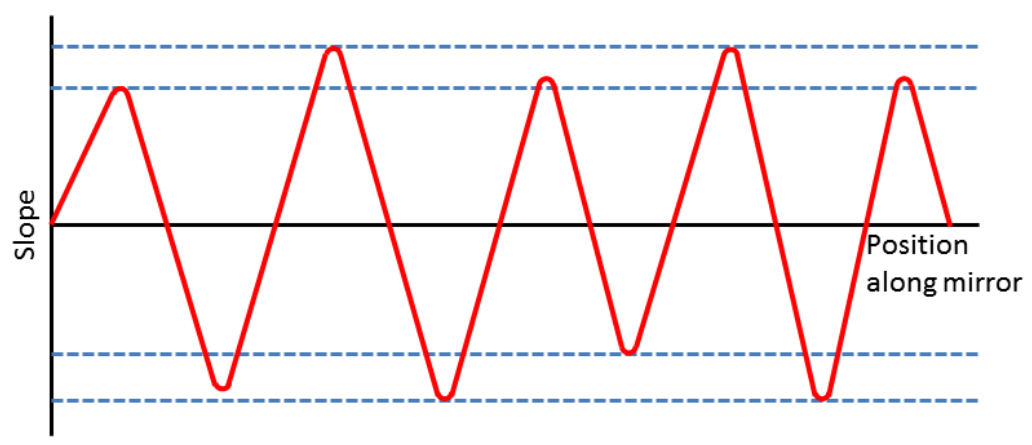

Figure 11: Schematic of re-entrant surface modification of continuous curvature set up to break up hot edges and ensure even spread of illumination across center of beam at focus. Shown with segments of equal length for simplicity but can be generalized to segments of unequal length.

\section{CONCLUSIONS}

To previous research on re-entrant surface modifications applied to a mirror for beam expansion, this article adds three innovations: the construction of re-entrant modifications on segments of any possible length and location, the deliberate choice of breakpoints to coincide with the position of the piezos and the figure errors of a real deformable bimorph mirror, and the application of well-suited re-entrant surface modifications to such a mirror. The beam produced at the focus by the simple concave modification has a shoulder and is breaking up into two more separate peaks. The fivesegment modification produces intense spikes at the edges but little other striation. The seven-segment modification produces spikes at evenly spaced intervals, and the main spikes are of similar size and lie in top of a similar background level. The striations that appear when a re-entrant modification is applied are caused mainly by the rounding of the curvature at the segment endpoints rather than by the random figure errors. This makes the performance of a re-entrant modification more predictable when applied to different deformable bimorph mirrors, especially for a bimorph of which the PRFs avoid overshoot, undershoot, or overlap. It also suggests ways in which the hot edges predicted by geometrical optics and observed in the data of this article might be reduced.

\section{ACKNOWLEDGMENTS}

Part of this work was undertaken on B16 at Diamond Light Source under proposals NT3803 and NT13515-1. The authors thank their colleagues at Diamond Light Source. David Laundy and Lucia Alianelli provided stimulating discussions, Ian Pape his support on B16, and Andrew Malandain his technical assistance. Thomas Sorensen and Juan Sánchez-Weatherby collaborated in the collection of the bimorph mirror PRFs of the vertical focusing mirror of beamline I02. Robin Owen and Danny Axford assisted in the examination of the vertical microfocusing mirror of their beamline I24.

\section{REFERENCES}

[1] Babcock, H. W., "The possibility of compensating astronomical seeing," Publications of the Astronomical Society of the Pacific 65 (386), 229-236 (1953).

[2] Susini, J., Labergerie, D. and Zhang, L., "Compact active/adaptive x-ray mirror: Bimorph piezoelectric flexible mirror," Rev. Sci. Instrum. 66 (2), 2229-2231 (1995).

[3] Signorato, R., Hignette, O. and Goulon, J., "Multi-segmented piezoelectric mirrors as active/adaptive optics components," J. Synchrotron Rad. 5 (3), 797-800 (1998).

[4] Signorato, R. and Ishikawa, T., "R\&D on third generation multi-segmented piezoelectric bimorph mirror substrates at Spring-8," Nucl. Instrum. Methods Phys. Res. A 467-468 (1), 271-274 (2001).

[5] Laundy, D., Alianelli, L., Sutter, J., Evans, G. and Sawhney, K., "Surface profiling of X-ray mirrors for shaping focused beams," Opt. Express 23 (2), 1576-1584 (2015).

[6] Sutter, J. P., Alcock, S. G., Rust, F., Wang, H. and Sawhney, K., "Structure in defocused beams of X-ray mirrors: causes and possible solutions," Proc. SPIE 9208, 92080G (2014). 
[7] Spiga, D., Raimondi, L., Svetina, C. and Zangrando, M., "X-ray beam shaping via deformable mirrors: Analytical computation of the required mirror profile," Nucl. Instrum. Methods Phys. Res. A 710, 125-130 (2013).

[8] Nicolas, J. and García, G., "Modulation of intensity in defocused beams," Proc. SPIE 8848, 884810 (2013).

[9] Laundy, D., Sawhney, K., Nistea, I., Alcock, S. G., Pape, I., Sutter, J., Alianelli, L. and Evans, G., "Development of a multi-lane X-ray mirror providing variable beam sizes," Rev. Sci. Instrum. 87 (5), 051802 (2016).

[10] Sutter, J. P., Alcock, S. G., Kashyap, Y., Nistea, I., Wang, H. and Sawhney, K., "Creating flat-top X-ray beams by applying surface profiles of alternating curvature to deformable piezo bimorph mirrors," J. Synchrotron Rad., DOI:10.1107/S1600577516013308, (November 2016).

[11] Math Works, Inc. Matlab Version 7.0.1.24704 (R14) Seriee Pack 1. Natick, Massachusetts, USA (2004

[12] Yamauchi, K., Mimura, H., Inagaki, K. and Mori, Y., "Figuring with subnanometer-level acracy by numerically controlled elastic emission machining," Rev. Sci. Instrum. 73 (11), 4028-4033 (2002).

[13] Sawhney, K., Alcock, S., Sutter, J., Berujon, S., Wang, H. and Signorato, R., "Characterization of a novel super-polished bimorph mirror," J. Phys.: Conf. Ser. 425 (5), 052026 (2013).

[14] Alcock, S. G., Sawhney, K. J. S., Scott, S., Pedersen, U., Walton, R., Siewert, F., Zeschke, T., Senf, F., Noll, T. and Lammert, H., "The Diamond-NOM: A non-contact profiler capable of characterizing optical figure error with sub-nanometre repeatability," Nucl. Instrum. Methods Phys. Res. A 616 (2-3), 224-228 (2010).

[15] Wang, H., Sutter, J. and Sawhney, K., "Advanced in situ metrology for x-ray beam shaping with super precision," Opt. Express 23 (2), 1605-1614 (2015).

[16] Sawhney, K. J. S., Dolbnya, I. P., Tiwari, M. K., Alianelli, L, Scott, S. M., Preece, G. M., Pedersen, U. K. and Walton, R. D., “A Test Beamline on Diamond Light Source,” AIP Conf. Proc. 1234, 387-390 (2010).

[17] Hignette, O., Freund, A. and Chinchio, E., "Incoherent X-ray mirror surface metrology ," Proc. SPIE 3152, 188199 (1997).

[18] Sutter, J. P., Alcock, S. G. and Sawhney, K. J. S., "Automated in-situ optimization of bimorph mirrors at Diamond Light Source,” Proc. SPIE 8139, 813906 (2011).

[19] Alcock, S. G., Sutter, J. P., Sawhney, K. J. S., Hall, D. R., McAuley, K. and Sorensen, T., "Bimorph mirrors: The Good, the Bad, and the Ugly," Nucl. Instrum. Methods Phys. Res. A 710, 87-92 (2013).

[20] Alcock, S. G., Nistea, I., Sutter, J. P., Sawhney, K., Fermé, J.-J., Thellièr, C. and Peverini, L., "Characterization of a next-generation piezo bimorph X-ray mirror for synchrotron beamlines," J. Synchrotron Rad. 22 (1), 10-15 (2015). 